\title{
Value-Driven Analysis of New Paradigms in Space Architectures: An Ilities-Based Approach
}

\author{
Dr. Daniel Hastings. ${ }^{1}$ \\ Massachusetts Institute of Technology, Cambridge, MA, 02139 \\ Capt. Paul La Tour, USAF ${ }^{2}$ \\ Massachusetts Institute of Technology, Cambridge, MA, 02139 \\ and \\ ENS Ben Putbrese, USN ${ }^{3}$ \\ Massachusetts Institute of Technology, Cambridge, MA, 02139
}

\begin{abstract}
Current commercial, civil, and military space architecture designs perform exquisitely and reliably. However, today's architecture paradigms are also characterized by expensive launches, large and expensive high-performance spacecraft, long development cycles, and wide variations in ground architectures. While current assets provide high-quality services, and future assets are slated to improve performance within the same design frameworks, proposed future architectures may not be capitalizing on technology improvements, system innovations, or policy alternatives explored during the last two decades. This paper identifies five "trends" along which space architectures may develop, aimed at granting systems several “ilities," such as resiliency, robustness, flexibility, scalability, and affordability. The trends examined include: commercialization of space, significant reductions in launch costs and the development of hybrid or reusable launch systems, development of on-orbit infrastructure and servicing, aggregation or disaggregation of orbital assets, and the automation and standardization of ground architectures. Further refinement of these key technological and system trends could result in major paradigm shifts in the development and fielding of space operations as well as lead to space architecture designs in the future that are radically different from those today. Within the framework of systems engineering ilities and risk management, this paper reviews current literature surrounding these new change trends and justifies their potential to cause significant paradigm shifts. By examining the work and research conducted so far through an ilities-based approach, systems engineers can more fully appreciate the value being offered by these trends.
\end{abstract}

\section{Introduction}

Cunel URRENT commercial, civil, and military space architectures perform exquisitely and reliably, with many space vehicles ranking among the most advanced and impressive technologies ever created. However, while space systems work quite well, the paradigms driving space architectures are also often characterized by increasingly expensive manufacturing and launch, long development cycles, and wide variations in ground architectures. Future

\footnotetext{
${ }^{1}$ Cecil and Ida Green Education Professor of Engineering Systems and Aeronautics and Astronautics, Massachusetts Institute of Technology, 77 Massachusetts Ave., Cambridge, MA 02139.

${ }^{2}$ PhD Candidate, MIT Engineering Systems Division, 77 Massachusetts Ave., Building E40-261, Cambridge, MA, 02139.

${ }^{3}$ SM Candidate, MIT Technology and Policy Program, 77 Massachusetts Ave., Building E40-369, Cambridge, MA, 02139 .
} 
assets and proposed architectures are largely slated to improve performance within these same design frameworks, and thus they may not be addressing long-term maintainability issues or capitalizing on technology improvements, system innovations, and policy alternatives explored during the last two decades. This paper identifies five "trends" along which space architectures may develop, aimed at making these systems more robust, resilient, responsive, and extensible as well as addressing core sustainability issues. The trends examined include: commercialization of space, significant reductions in launch costs and the development of hybrid or reusable launch systems, development of on-orbit infrastructure and servicing, aggregation or disaggregation of orbital assets, and more standardized and automated ground systems. Changing sociotechnical realities are beginning to increase the desire for and economic feasibility of such concepts and infrastructure alternatives. Furthermore, refinement of these key technological and system trends could set cause lasting paradigm shifts in the development and fielding of space operations as well as lead to space architecture designs in the next few decades which are radically different from those today.

As a launching point for analyzing the effects that these five trends could have on space architectures in the upcoming decades, this paper begins with a review of current literature, in order to inform readers of the research and analysis which has already been applied to these potentially paradigm-changing concepts and technologies. Each trend (commercialization of space, launch cost reductions and new vehicle development, on-orbit infrastructure and servicing, aggregation/disaggregation, and more automated and standardized ground architectures) is reviewed in depth and major works are presented. Throughout the literature review, the authors also outline and defend their justifications for the characterization of each trend as a key paradigm-changer.

Next, the authors align these trends with select "ilities," based on MIT SEAri's Value-Driven Engineering and Architecting practices, in order to create a framework for discussion. The authors contend that current space systems and their architectures strongly exhibit capability, reliability, durability, and quality, but are often found lacking in terms of robustness, resiliency, scalability, extensibility, testability, flexibility, and survivability. These shortfalls of agility are historically balanced with the constraints of perceived affordability; i.e., while all of these characteristics and ilities are desired, they are almost always cost-limited and therefore remain largely unpursued. Furthermore, the difficulty of quantifying the value of agility (and the subsets of agility) in a system often causes systems engineers and decision-makers to downplay this value when compared to more tangible performance parameters. Thus, this paper argues that it is the goal of each of the five identified trends, either individually or in conjunction, to add or improve these ilities in space architectures while also maintaining or even decreasing costs.

Along with the discussion of ilities, concepts of risk management within space architectures are reviewed, with the main hypothesis being that the implementation of these new trends would make fundamental changes to how risk management of space architectures is conducted. The trends of reduced launch costs, on-orbit infrastructure and servicing, and disaggregation in particular should lead systems engineers to accept greater risk in fielding new architectures. This paper also theorizes that these trends can begin to counteract the reinforcing feedback loop of increasing launch costs and lengthening spacecraft development cycles. Further areas of research and analysis will be identified in the conclusion.

\section{A. Trends in space architecture design}

\section{Background}

Historical trends in architecture designs have been largely driven by technological improvements and increasing sophistication of space vehicles. Since the first satellites were launched into space in the 1950's and 60's, spacecraft have consistently become more massive, powerful, and enduring. Many geosynchronous communications satellites launched during the last decade weigh around $2,000 \mathrm{~kg}$, operate near $10,000 \mathrm{~W}$ of power, and last at least 12 years. ${ }^{1}$ Indeed, these large, powerful, long-lasting space vehicles have largely become the norm for big-ticket space projects. However, architecture designs are increasingly lagging behind the rapid development of new technologies, and it is often found that architectures designed for the economic and technological realities at the time of launch are not adequate for the actual present-day environment. ${ }^{1}$

Large monolithic space vehicles — while highly sophisticated and reliable-have become characterized by increasing costs and lengthening development timelines, in addition to increasing launch and insurance costs. Furthermore, typical long design lifetimes may contribute to the obsolescence of onboard technology and the underutilization of increasingly rapid technological advances. Many have questioned the long-term viability of the current paradigm in architecture design and instead point to smaller, more responsive constellations of disaggregated spacecraft that take advantage of increased miniaturization of required technologies. This concept is also endorsed by military architecture designers, due to its inherent survivability against enemy attack or interference. ${ }^{2}$ 


\section{B. Comparison of commercial, civil, and defense space architectures}

The three major categories of space systems architectures—commercial, civil, and defense-are all characterized by relatively unique requirements and capabilities, in addition to the general desire for reliability and cost effectiveness. For commercial entities, the main driver of architecture design is profit maximization and reliable service to the customer. Some commercial space entities are also strongly linked to public agencies, providing launch services, communications, some on-orbit infrastructure, etc., and thus may be strongly reliant on government contracts or subsidies. As technologies advance and new business models are tested, commercial firms are beginning to explore new architectures, such as those required for terrestrial remote sensing and space tourism. ${ }^{3}$

Civil space systems lack the explicit need to turn a profit on their space activities, but are still held accountable by policymakers and taxpayers' representatives in government to provide useful returns on investment. Therefore, civil architectures are often devoted to furthering scientific exploration and providing the on-orbit infrastructure necessary for all actors to maintain robust space activities; i.e., those activities which may not be profitable enough to be provided by commercial entities. Civil systems like many of those launched and operated by NASA and NOAA are also used to provide valuable services to organizations and individuals, such as space weather systems and terrestrial imaging. Furthermore, civil space architectures may be used to test new technologies and operational concepts for overall viability before transitioning successful projects or capabilities over to commercial users. ${ }^{3}$

Finally, Department of Defense (DoD) space architectures share several similarities with civil systems but with the additional desire for increased survivability and resiliency against a much larger spectrum of outside threats. This often leads to greater overall requirements from defense architectures than from commercial or civil. Also, their missions are typically viewed as vital to national security, thereby increasing overall infrastructure needs and costs. ${ }^{3}$ Recently, DoD architectures have been characterized by increasing costs and lengthening development cycles, and thus there is now greater focus on responsiveness and increased cooperation with other entities in an effort to drive down costs and development lags; for instance, through the hosting of less vital missions on board commercial satellite busses. ${ }^{2}$ In addition, a new thrust for increased resiliency and disaggregation of space architectures has emerged, especially from the U.S. Air Force Space Command, in order to increase overall survivability, responsiveness, and flexiblity while also decreasing costs. ${ }^{4}$ One final note concerning DoD architectures is that, similar to civil systems, military space can also serve as a testbed for the development of new technologies before transitioning them to commercial or civil use, an example of which would be the opening of the GPS signal to users across the globe.

\section{Commercialization of space}

Increased commercialization of space activities is a growing trend that seeks to leverage the strengths of industry in order to decrease costs and maintain quality for all types of space architectures. This trend does not necessarily reflect a change in technology, but rather a shift in policy that seeks to push some of the risks and costs of space architectures to commercial, profit-seeking firms. Many actors and stakeholders foresee significant benefits from increased commercialization of space, including government agencies attempting to drive down costs and entrepreneurs seeking to establish new business models for space launch and operations. The background that follows is a review of much of the work currently being done to drive this new developmental and operational paradigm.

Anderson provides an extensive overview of the benefits of shifting more responsibility for spaceflight and technology development over to commercial entities. He asserts that the public versus private argument is a "false dichotomy," and that the greatest benefits lie not in choosing one over the other but in partnerships. Such alliances allow both parties to leverage their core competencies and complement each other's strengths; for example, by paying companies like SpaceX to perform more mundane tasks like LEO payload delivery and ISS resupply, NASA can then direct more funds to deep-space science and exploration activities that are exciting but lack a clear profit motive. Private firms have also demonstrated greater efficiency and cost reductions than government programs, primarily through such proven concepts as learning curves, standardization of manufacturing practices, and more efficient industrial pipelines than those often found in programs with heavier government oversight. In fact, one NASA report estimated that SpaceX's Falcon 9 launch vehicle was developed for a third of the cost that would have been required of a public program. ${ }^{5}$

Furthermore, NASA has found great success in its new Space Act Agreements, in which NASA has agreed to pay private spaceflight firms in increments as they achieve set milestones in technology development and operational readiness. This is a far more advantageous arrangement than more traditional "cost-plus" contracts, which simply pay a contracted firm the cost of the project plus a pre-determined percentage of the cost on top. While the partnerships forged under Space Act Agreements have yet to come fully to fruition, it is already believed 
that they are largely successful in avoiding the cost overruns and delayed development cycles often encountered under cost-plus arrangements. ${ }^{5}$

Next, Gong et. al. describe the Commercially Hosted Infra Red Payload (CHIRP) program, which was conceived as a means of transitioning space architectures towards commercial partnerships while also decreasing transition risks. Recent trends in the commercial space industry have led to more mature commercial satellite busses that can facilitate the inclusion of simpler, smaller, and less expensive payloads in addition to the main payload of the commercial spacecraft. The CHIRP program tested the idea that commercially hosted payloads could assist in achieving the end goals of affordability, resiliency, and capability, and the authors outline the preliminary evidence that hosted payloads are a reliable and affordable method for increasing resiliency of space assets. It is also believed that hosted payloads can leverage the competition between commercial spacecraft manufacturers and take advantage of the stable production rates of commercial busses in order to lower costs and increase efficiency. Furthermore, future catalysts to facilitate the hosted payload concept include technology advances in commercial busses, smaller, simpler payloads, more freedom to reshape commercial missions to better match payload needs, and the ability to implement more streamlined rapid acquisition approaches. ${ }^{6}$

It should also be remembered that the hosted payload concept does not work exclusively to the benefit of military and civil entities looking for open space aboard commercial vehicles. The private firms hosting others' payloads also benefit from the increased revenue and long-term contracts which would accompany an increase in commercial payload hosting (if not, there is little chance that private actors would agree to sharing the power and physical space aboard their vehicles). Hosted payloads would also act as an additional means of strengthening the space industrial base by shifting a larger portion of the manufacture and development of spacecraft busses to the commercial sector. Thus, this concept theoretically comprises a mutually beneficial partnership between commercial and civil/military space architectures. ${ }^{6}$

Finally, another area of commercial innovation involves several new start-ups which are testing new means of turning a profit in space. Companies like SkyBox, PlanetLabs, and UrtheCast have all launched or are planning to launch imagery payloads and then sell their high quality images and data to governments and businesses. ${ }^{7}$ In addition, Virgin Galactic is nearing completion of its WhiteKnightTwo and SpaceShipTwo reusable launch vehicle combination, which seek to gain technological maturity as a space tourism platform before transitioning to the responsive launch of small low Earth orbit payloads. It should also be noted that Virgin Galactic has already accepted payment from over 650 passengers to help bankroll not only its tourism business but also further commercial space projects. ${ }^{8}$

\section{Launch cost reductions and the development of hybrid or reusable launch systems}

Ever-increasing launch costs have long been a dominant feature in the development and fielding of space systems, and few solutions to this dilemma have taken hold. One potential solution which has been consistently discussed over the last few decades is the reusable launch vehicle (RLV). Rasky et. al. examine worldwide experiences with RLV's and identified the primary economic challenges faced by RLV's as opposed to expendable launch vehicles. They also analyze RLV feasibility according to simple economic practices, primarily return on investment calculations, and identify the critical link between high flight rate and economic viability. They find that in order to compete with expendable launch vehicles, RLV's must have a significantly higher flight rate-higher by at least a factor of 10 per year. ${ }^{9}$ It should be remembered that the most experienced RLV, the Space Shuttle, not only did not live up to its expectations as a more cost-efficient transport to orbit but also had an almost $50 \%$ reduction in payload-to-orbit as compared to common ELV's. Furthermore, to match the ELV's rate of return, partially reusable launch vehicles (PRLV's) must have a yearly flight rate of about 3, RLV's must have a flight rate of about 10, and a highly reusable launch vehicle (HRLV, such as the original concept for the Space Shuttle) must launch about 39 times per year, to account for the operations and maintenance costs. ${ }^{9}$

Rasky et. al. also estimate that for the Space Shuttle to have been competitive with ELV's, it would have required a flight rate of about 15 per year per vehicle, or 45 flights per year for a Shuttle fleet of three vehicles (the Shuttle's best operational year had a flight rate of approximately 1.5). ${ }^{9}$ Understandably, the current launch market is oriented to ELV's, and the authors recommend a combined market and technology development approach, such as that being pursued by NASA's Commercial Orbital Transportation Services (COTS) program, in order to bring RLV's and HLV's to an economically feasible and competitive status.

Next, in a jointly sponsored study by DARPA and NASA, Bartolotta et. al. explore the trade space of horizontal launch system concepts through an exhaustive literature review of the past 70 years. Their Horizontal Launch Study identified potential near- and mid-term concepts capable of delivering 15,000 lb. payloads to a $28.5^{\circ}$ due East inclination, 100 nautical-mile low-Earth orbit. RDT\&E costs for a new Earth-to-orbit technology demonstrator are estimated to be approximately $\$ 10$ billion in 2010 dollars for a new subsonic carrier aircraft, and as high as $\$ 17$

4

American Institute of Aeronautics and Astronautics 
billion for a new supersonic and $\$ 25$ billion for a new hypersonic carrier aircraft. ${ }^{10}$ Amortized over each launch, this adds between $\$ 6,000$ and $\$ 13,000$ per pound of payload and soon overwhelms all other costs. Thus, the authors recommend that if the government decides to pursue horizontal launch they should allow commercial firms to bear the largest burden of cost and risk. Bartolotta et. al. believe that a modified existing subsonic carrier aircraft with a liquid propellant launch vehicle could carry a payload up to $20,000 \mathrm{lb}$. at a cost of $\$ 8,860 \mathrm{per} \mathrm{lb}$., with overall RDT\&E costs estimated at less than $\$ 2$ billion if using current technology and partnering with commercial entities. ${ }^{10}$ In their view, horizontal launch is a competitive alternative to expendable launch vehicles for LEO missions that value responsiveness and flexibility. ${ }^{10}$

While all of the work described above may not seem particularly hopeful with regards to the prospects of a viable, less expensive launch capability, there have been more concrete developments along this trend in private industry. For example, SpaceX’s most current pricing for its Falcon 9 launch vehicle is \$56.5 million, which translates to $\$ 4,296$ per kilogram delivered to low Earth orbit—far less than the typically $\$ 10,000+$ per kilogram that space systems engineers have grown accustomed to. ${ }^{11}$ SpaceX has also had several successful launches and is continuously evolving its rocket catalogue to develop more powerful and cost-effective launch vehicles. In addition, Virgin Galactic is nearing operational capability of its own reusable launch system, aiming for its first commercial operations later this year. ${ }^{8}$ If companies like SpaceX and Virgin Galactic continue along this trend of succesful and much less expensive expendable and reusable launch, it could cause major shifts in how space systems engineers design long-term space architectures.

\section{E. On-orbit servicing and infrastructure}

The ability to repair, refuel, and reposition spacecraft in orbit has often been proposed as a means of radically restructuring the conduct and planning of space missions. Accordingly, several studies have been conducted to explore the feasibility of such a capability. In his thesis, Richards developed a methodology to assess serviceability across the four activities of rendezvous, acquire, access, and service, and he estimates that there are approximately 25 servicing opportunities for GEO spacecraft each year. ${ }^{12}$ He also finds that the main challenges to implementing more OOS are not technical but social and political — that is, the technology must be proven safe and reliable before it receives wider implementation, leading to a "chicken-and-egg" problem of maturing the technology when few wish to undertake the required funding and development. ${ }^{12}$

Next, according to Long et. al., on-orbit servicing of satellites appears to be technically feasible but may not yet be economically feasible, and a new value proposition for satellite architectures must therefore be developed. This new value proposition is oriented towards rapid response to technological or market change and design of satellites with less redundancy; in other words, satellites must be designed with OOS explicitly in mind. Long et. al. find that 130 servicing tickets of various urgency could be expected to be generated over a 5 year period by the set of 335 GEO satellites, but that the economic feasibility of on-orbit servicing remains questionable. ${ }^{13}$ The current generation of spacecraft is too reliable to ensure market share for OOS, and a paradigm shift to GEO spacecraft with shorter lifetimes may be required. New value propositions must be incorporated into the design and operation of satellites, and OOS must be able to provide large performance gains over the long design lifetimes of satellites. Also, if customers begin to more heavily value flexibility and responsiveness in their space operations, then OOS could capitalize on the shifting paradigm. ${ }^{13}$

In describing the rationale for the need of OOS capabilities, Benedict describes how NASA and DARPA are executing robotic programs for in-orbit servicing, but potential users have been hesitant to embrace this technology. Servicing missions could include refueling, towing, rescue of satellites which have suffered launch shortfalls or orbital anomalies, and even the removal of orbital debris. According to Benedict, though, the space industry is conservative and will not embrace on-orbit servicing until organizations like NASA and DARPA prove it is feasible. Then, demand for missions is expected to greatly increase. There are several cases where it may be less expensive and more efficient to service a satellite than to replace it. For example, if a commercial communications satellite at GEO requires an investment of $\$ 200-\$ 400$ million, most firms would pay a sum below or even equal to that initial investment in order to save a satellite from an otherwise mission-ending anomaly. The case for refueling or servicing well-functioning spacecraft is more difficult to sell, though, as firms often prefer replacement of the spacecraft with a more technologically capable one, rather than service of their current asset. ${ }^{14}$

If nothing else, one obvious example of the benefits of on-orbit servicing is provided by the Hubble Space Telescope, which underwent five separate servicing missions to fix and improve many aspects of this well-known and long-lived spacecraft. The fact that the Hubble Telescope had been designed specifically with servicing in mind greatly aided in the success of all of these missions, as components could be easily accessed and modified by astronauts. While cost reductions and overall affordability were not a main driver in these missions, they still speak to the much greater flexibility and maintainability that can be provided through OOS. ${ }^{15}$ 
Finally, with regards to new concepts in infrastructure, Golkar et. al. have performed extensive research into the hosted, disaggregated space infrastructure concept within his Federated Satellite Systems (FSS) framework. ${ }^{16}$ The FSS framework integrates the study of technical feasibility, market assessment, and business case proposition validation of FSS terminals mounted as payloads on hosting spacecraft. FSS allows spacecraft to share in-orbit resources in an opportunistic fashion. Examples of in-orbit resources include processing power, link capacity, and data storage. Commercial implementations of the FSS concept are designed to create in-orbit markets of space resource commodities, and to enhance sustainability, cost-effectiveness, robustness, and reliability of participating missions. Also, one key test bed that Golkar identifies for the implementation of an orbital infrastructure is the International Space Station, as it has all the necessary power and hardware needed to test new infrastructure technologies (Golkar, 2013). ${ }^{17}$ Optical hosted payloads are identified as one of Golkar's preferred solutions, as they provide simpler, lighter, and more effective data relay and processing systems when compared to RF. ${ }^{18}$ The ISS as a hosted payload hub could create a market of millions to hundreds of millions in Euros for optical communications and data processing, and potential markets of interest would be those LEO spacecraft in sun-synchronous orbits conducting Earth observation and telecommunications missions. The FSS concept would also make use of the unused telecommunications capacity available in participating spacecraft at any given time.

\section{F. Aggregation or disaggregation of orbital assets}

Trends in aggregation and disaggregation of space vehicles are essentially a spectrum; that is, both represent changes in architecture design, either towards a consolidation of missions on to fewer platforms or the fractionation of a mission across several smaller, simpler vehicles. Aggregation is an especially salient concept in the face of rising launch and development costs, as designers may seek to decrease overall costs by adding new components to an existing platform and then using only one or a few launches to accomplish several missions. While aggregation of assets is an acknowledged trend in space architectures, it is not viewed as a particularly paradigm-shifting one, and thus the majority of this section focuses on the benefits that could potentially result from a shift to the other end of this spectrum: disaggregation.

The fractionated spacecraft architecture is a much-discussed but not yet widely implemented concept for future space missions. Department of Defense planners like this concept because of the inherent survivability of a greater number of orbital targets, ${ }^{4}$ while civil and commercial firms may seek greater flexibility in space architectures and possible cost reductions resulting from smaller, simpler satellites. Mathieu finds that the main goal of the fractionated spacecraft concept is to have an infrastructure of standardized modules that could support various payloads and would be composed of several smaller, simpler modules that could be more easily replaced, greatly increasing the flexibility and overall responsiveness of the constellation. The first conclusion of her thesis is that at a given performance level, utility significantly increases with fractionation over an equivalent traditional architecture. ${ }^{19}$ Furthermore, while fractionated spacecraft are almost always more expensive than traditional ones, the infrastructure associated with fractionated spacecraft often is not (depending on infrastructure design parameters), and thus fractionated systems can dominate traditional ones in terms of both cost and utility. Therefore, if flexibility is valued enough, customers will choose fractionated spacecraft over traditional ones. However, the aerospace industry in its current state is not structured for the implementation of fractionated spacecraft, and as such she concludes that the government should impose policies and incentives which will enable the shift. ${ }^{19}$

In addition, Mathieu and Weigel assessed fractionated spacecraft in terms of the following attributes: mass, maintainability, scalability, flexibility and responsiveness (cost was also considered as an independent variable). Using worst-case scenario estimations, they found that mass and cost penalties are significant when shifting from traditional to fractionated spacecraft. However, maintainability, scalability, flexibility, and responsiveness all improve with fractionation, and they conclude that if these attributes are valued enough, then the advantage quickly shifts to fractionated spacecraft. ${ }^{20}$

Next, Brown and Eremenko apply a value-centric approach to the fractionation of space architectures in their discussion of DARPA's F6 program. From their experiences in this program, the authors found that disaggregated architectures offer greater flexibility, robustness, and scalabilty over more traditional monolithic space vehicles, and if stakeholders are sufficiently convinced of the value of such attributes in a space architecture then they will accept the costs and risks associated with switching to the fractionated paradigm. Furthermore, Brown and Eremenko discuss the positive industrial base effects that could result from a more disaggregated operational paradigm, including reduced barriers to entry, increased number of competitive opportunities, and increased use of small responsive launch vehicles. Overall, the authors put forward a strong case that a more value-based systems engineering approach to space architectures ultimately favors the fractionated spacecraft paradigm. ${ }^{21}$

Finally, Sondecker and La Tour describe their extensive experience working with USAF CubeSats. Advances in CubeSat technology are believed to hold the potential to allow the defense space acquisition community to rapidly 
procure and deploy capable satellite systems at substantially reduced cost. Small satellites no longer equate to reduced capabilities, and using COTS components leads to costs that are even further reduced while also increasing overall efficiency in acquisition and development. Efficiency can be further increased by maturing ground systems and employing concepts like the Common Ground Architecture (discussed below), and timelines can be shortened if CubeSats are well-engineered to withstand the increased loads of ride-share missions. ${ }^{22}$ Furthermore, smaller satellites can offload some missions from larger programs where aperture and power requirements are not as high, and they can also serve as technology maturation and risk reduction testbeds for larger programs. This research provides greater credibility to the disaggregation concept due to its demonstration that small satellites have reached great sophistication and can provide many of the capabilities of much larger monolithic space vehicles at reduced cost. $^{23}$

\section{G. Automation and standardization of ground architectures}

The most capable and well-documented ground system known to the authors is the Air Force Satellite Control Network (AFSCN). Currently comprised of nine ground stations spread across the globe that handle over 400 satellite passes per day and achieve an impressive 99\% satisfaction of contacts, the AFSCN has grown significantly over the past 30 years, adding on new capabilities as needed. ${ }^{24}$ However, it currently takes two years to add a new satellite mission to the AFSCN network, ${ }^{24}$ which fortunately remains in line with the typical development timelines for satellites. AFSCN also relies heavily on well-trained and disciplined personnel to compute each 24-hour set of passes and plan the next day's operations in multiple planning cells. While AFSCN's process has proven capable, like other aspects of space it has achieved this capability through a great deal of money and labor. The current AFSCN architecture will not scale indefinitely, though. Eventually humans will be overwhelmed by this NPcomplete tradespace, as assigning more and more passes and de-conflicting an ever-growing number of possible solutions will become too great a task for human operators. As such, more automated ground operations will be essential as the number of on orbit assets increases. ${ }^{25}$

A well-documented and possible solution to some AFSCN challenges may be the U.S. Navy's Common Ground Architecture (CGA), also known as the Neptune System. To date, CGA has at most handled tens of satellites at once, far less than the AFSCN, but it is designed with scalability in mind. ${ }^{26}$ For instance, CGA (like AFSCN) uses rules to assign different satellites different priorities or weightings for resource allocation. Pass satisfaction and contact duration are based upon a hierarchy to determined resource allotment for mission requirements and sharing of resources. CGA differs in that after the input of each satellite into its database, it uses a logic engine/solver to automatically compute a best solution. This logic engine extends beyond just the satellites by also selecting the hardware to service each request at each site, which enables optimal use of resources across the globe and at each satellite farm. It also positions CGA to recompute all passes for a 24 hour period within seconds, whereas with human operators there is almost no contingency capability and missed passes will likely be lost or need to be made up the next day (creating a cascade of issues). Standing up a new CGA site is also a strength of this system. Each "rack" comes with the capability to service around 100 satellites, and each is designed for smooth integration into the overall architecture. ${ }^{26}$

The CGA software has also aided the rise of operationally relevant CubeSats. It serves as a commercial-off-theshelf (COTS) available rack that can be used with a variety of antennas to provide communications when and where they are needed. At a cost point below $\$ 150 \mathrm{~K}$ for a full setup of the Mobile CubeSat Command and Control system (MC3), CGA is a low cost option for research and development of specific components. ${ }^{26}$ Ultimately, this type of automated ground system also improves testability of future systems, as it enables on-orbit testing at substantially lower cost. Based on CGA's use of databases for command and telemetry, a new mission can be added just by adding a new database; in other words, there is no need to hardcode anything when adding a new mission, leading to substantially faster and cheaper integration of new components. While CubeSats are currently limited to a small class of missions, their increasingly high degree of complexity has proven a good proxy for tackling some of the issues associated with large constellations, as the work required from the ground station is nearly identical.

\section{H. Risk management and effects on architecture cost and development}

NASA procedural requirements currently define risk management as "a set of activities aimed at achieving success by proactively risk-informing the selection of decision alternatives and then managing the implementation risks associated with the selected alternative., ${ }^{27}$ While always a concern, the historical trend in risk management of space systems has been characterized by increases in system reliability, coupled with increasingly high levels of risk aversion. This is not surprising, as the early days of trial, error, and experimentation of new space systems have long since given way to highly reliable and advanced systems which are nevertheless constantly haunted by the small possibility of a catastrophic system failure. Indeed, Szajnfarber, Richards, and Weigel identify space systems as 
being quite different from other emerging engineering systems, as the massive upfront costs of launch and development, as well as the near impossibility of correcting a spacecraft's failures after launch, have largely inhibited the development of an entrepreneurial spirit or a greater acceptance of risk when developing new technologies. ${ }^{28}$ Furthermore, the unbending requirement for success from every launch, combined with the extremely visible nature of space systems failures, has largely driven space architectures from an era of short-lived, single payload spacecraft to large, multi-payload, long-lived assets that require at least 5 to 10 years of development time. $^{28}$

One common technique for evaluating the risks involved with operating a new space system is to aggregate the probabilities of failure for all components of the system into a few common system failure probabilities. ${ }^{29}$ While such a worst-case scenario perspective on system failure probabilities can indeed help in decision-making during development, such an approach can also have the adverse side effect of unnecessarily restricting the use of new and relatively unproven concepts and technologies. As Murman, Walton, and Rebentisch point out, "risk and value are intimately interrelated, as the quality of the value metric is related to the probability of certainty of its representation." ${ }^{30}$ Thus, while there is inherent risk tied to discovering the value of such new concepts as commercialization, disaggregation, and on-orbit servicing and infrastructure, it should also be remembered that such concepts have the potential to bring unprecedented value to space architectures and may require new ways of evaluating risk. Indeed, all of the paradigm-changing trends identified are in some way aimed at reducing the influence of an increasingly risk-averse industrial culture on space systems development, which would eventually uncover full system value to customers and stakeholders.

One fundamental way in which these trends may alter current perspectives on risk management would be a shift towards a focus on availability of a service, rather than the simple presence of a capability. In other words, the space industry would begin to evaluate architectures' risk-posturing based on the risk of a service losing a percentage of its "uptime," with less emphasis placed on the health of each individual asset. For example, if an architecture is being designed for "graceful degradation" (see discussion of this concept below), then a constellation of disaggregated platforms would likely be more capable of maintaining performance throughout a perturbation than one large monolithic spacecraft, even if a few of the disaggregated platforms are taken out of service. This illustrates the way in which basic risk-posturing can be shifted from the risk of losing an asset to the risk of degraded performance and decreased delivery of a service, and it is the authors' opinion that each of the five trends discussed in this paper would directly drive this shift in risk management paradigms.

\section{Methods}

\section{A. Ilities}

Many authors have used ilities and similar value-defining attributes to describe the benefits of paradigmchanging trends. However, the definitions tend to vary across authors and publications. Therefore, in order to proceed with discussion and evaluation, it is necessary to clearly define the meaning behind each ility and therefore the value that each new trend would bring to the tradespace.

According to de Weck, Roos, and Magee, "The ilities are desired properties of systems, such as flexibility or maintainability (usually but not always ending in "ility"), that often manifest themselves after a system has been put to its initial use. These properties are not the primary functional requirements of a system's performance, but typically concern wider system impacts with respect to time and stakeholders than are embodied in those primary functional requirements. The ilities do not include factors that are always present, including size and weight (even if these are described using a word that ends in "ility")". ${ }^{31}$ Ilities are therefore the relatively intangible and often-leftunquantifed attributes of an engineering system which characterize the system's positive or negative response to changes in context encountered throughout its operation. If an engineering system operated in a perfectly static, unchanging environment throughout its lifetime, then the ilities could understandably be neglected during the design and development of the system, but of course such an environment has never existed outside of a classroom. Indeed, one could easily argue that context shifts now occur more frequently and rapidly than ever before, given the increasing pace of worldwide technological development. ${ }^{32}$

In addition, the definition of "context" is also key to understanding the importance and necessity of designing for ilities. Ross defines a system's context as "not only its operational environment, but also stakeholder sets, the expectations of the system, the resources available to the system, the competition for the system, and any other exogenous factors that affect the perceived value of the system." 33 While most often used in epoch-era analysis, the nature and implications of context shifts are nevertheless vital to any analysis of a system's ilities and its ability to adapt to new conditions throughout its lifetime. One example of a system which has seen a major context shift in 
the last few decades is the Global Positioning System, which shifted from an exclusively military system to a complete integration into the global economy.

With the overarching framework of ilities and context shifts defined, one must next understand the specific system traits and attributes described by each of the pertinent space architecture ilities:

\begin{tabular}{|c|c|}
\hline \multicolumn{2}{|r|}{ Definitions of Key Space Systems Ilities } \\
\hline Quality & The system is well made to achieve its desired function ${ }^{31}$ \\
\hline Reliability & The system operates throughout its lifecycle without need of repair or intensive maintenance ${ }^{31}$ \\
\hline Affordability & $\begin{array}{l}\text { The system remains cost effective in delivering value to the customer and relevant stakeholders } \\
\text { throughout its lifecycle and in the face of context shifts }\end{array}$ \\
\hline Survivability & The system minimizes the impacts of a finite-duration disturbance on overall performance ${ }^{35}$ \\
\hline Robustness & The system maintains an acceptable level of performance through context shifts throughout its lifecycle ${ }^{36}$ \\
\hline Resiliency & $\begin{array}{l}\text { The system can continue to provide required capabilities in the face of catastrophic failures, such as } \\
\text { system malfunctions, environmental challenges, or adversarial actions }{ }^{4}\end{array}$ \\
\hline Scalability & The system can be increased in scale without a penalizing increase in system complexity ${ }^{32}$ \\
\hline Extensibility & The system can incorporate new features and capabilities after beginning its lifecycle ${ }^{36}$ \\
\hline Testability & The system can be easily and realistically examined for faults prior to its operational lifecycle ${ }^{37}$ \\
\hline Flexibility & $\begin{array}{l}\text { The system's dynamic ability to take advantage of emergent opportunity and to mitigate risk by enabling } \\
\text { the system to respond to context shifts in order to retain or increase performance }{ }^{38}\end{array}$ \\
\hline
\end{tabular}

There are certain ilities which describe the response to similar issues faced by systems during their lifecycles, and in colloquial speech many of these ilities are often thought of as synomyns for one another. However, in order to avoid confusion and provide a thorough evaluation, each ility must be restricted to a rigid and tightly-formed definition. For instance, reliability refers to the system's ability to perform its desired functions with minimal to no repair or maintenance throughout its lifetime (much like a well-made automobile capable of travelling hundreds of thousands of miles), while robustness, on the other hand, refers to the system's continued output throughout context shifts during its lifetime (alluding to the previous example, think of the automobile continuing to function well as a family's main vehicle even as the family moves and grows). Similarly, survivability refers to the system's ability to minimize the impacts of a finite-duration disturbance, while resiliency is a system's ability to continue to perform after withstanding a catastrophic event (for instance, an automobile continues to handle well while driving over an icy patch [survivability] as opposed to the ability to continue driving after an accident [resiliency]). Furthermore, survivability may be thought of as continued acceptable performance even when the system is degraded by some external finite disturbance, while resiliency requires the system to undergo and recover from a catastrophic failure which would have otherwise brought about a complete end to system utility. While seemingly very similar, each ility describes a different means of responding to context shifts encountered over a system's lifecyle.

One other term which is often found associated with several of the ilities described above is graceful degradation, in which a system is described as continuing to provide significant utility even as the system begins to lose functionality over its lifecycle. This definition is closest in form to that of robustness, as it concerns a system's entire lifecycle. However, sometimes this term is also used to describe a situation more akin to survivability, in which the system is disrupted by some short-term perturbation but due to prior planning and integrated mechanisms the system can continue to output utility under duress. Thus it is important to discern whether graceful degradation is being applied to a system's performance over a full lifecycle or during a short-term disturbance, and the authors take care to draw this distinction whenever necessary.

Another key point with regards to the definitions provided above is that quality is the ility of performance-that is, quality speaks to the fact that there are varying degrees of performance, and just because a system can meet minimum function parameters does not mean it is delivering quality. de Weck et. al. make this clear when they discuss the importance of an engineering system not simply "working," but instead providing the maximum possible 
performance and utility to the customers and stakeholders. ${ }^{31}$ In addition, affordability is a distinct attribute from lifecycle costs and net present value calculations, as this ility describes a system's more intangible ability to remain cost-effective throughout its lifecycle, even in the face of context shifts and changing customer needs. If a system requires costly alterations to continue to deliver value after a context shift, then it is clearly lacking in affordability. It should be noted that the trends identified in this paper affect both the affordablity of a space system throughout context shifts and the overall lifecyle costs of a system, but these two attributes (affordability and reduced lifecycle costs) are distinct—reduced costs being an added benefit that are derived more through sound business practices, such as learning curves, competition, better continuity, an educated workforce, etc.

Finally, one system attribute which is often discussed, especially in DoD space architecture design, is responsiveness. The U.S. Air Force Operationally Responsive Space Office defines its mission as "to plan and prepare for the rapid development of highly responsive space capabilities that enable delivery of timely warfighting effects and, when directed, develop and support deployment and operations of these capabilities to enhance and assure support to Joint Force Commanders' and other users' needs for on-demand space support, augmentation, and reconstitution." ${ }^{39}$ While responsiveness does not itself receive a tighter definition, it aligns most closely with flexibility, as both are concerned with timely adaptation and continued performance throughout context shifts.

\section{Discussion}

With the framework established in the background and methods above, the true significance of the five identified trends-commercialization, launch reductions and reusable launch vehicles, on-orbit servicing and infrastructure, disaggregation and aggregation, and automated ground architecture-can now be explored. In order to justify each trend as a paradigm-changing technology or concept, the major sought-after ilities associated with each are listed and discussed. In addition, the main components of risk that each trend will likely mitigate are analyzed, as it is the reduction of certain risks in conjunction with newfound ilities which will actually drive new paradigms. Finally, each individual trend will primarily affect different phases of systems' lifecycles, as well as alter the spending profile and allocation of funds throughout architectures' development and operations. As a point of reference, the Department of Defense acquisition framework will be used to describe the phases of a system's lifecycle.

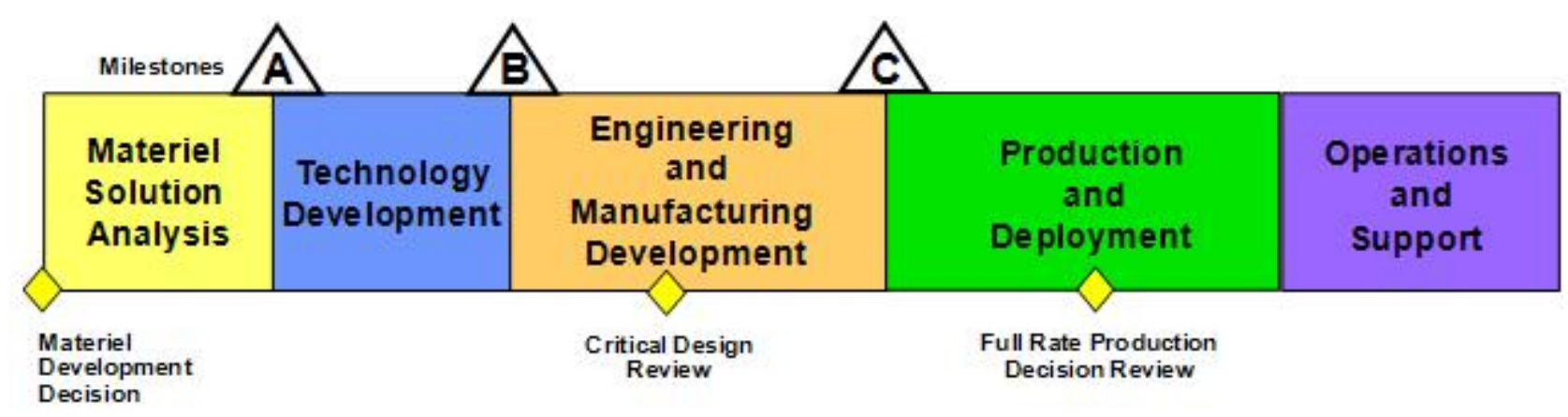

Figure 1. This framework illustrates the stages of DoD technology development and acquisition. ${ }^{40}$

\section{A. Commercialization of space}

Commercialization of space activities is not a change in technology but rather a change in policies and the ways in which public and private entities interact. The commercialization concept is directed at decreasing costs of specific activities while maintaining quality, and it does this by leveraging what industry does well, such as establishing efficient manufacturing pipelines and reaping the benefits of learning curve effects (proven in many other private commercial ventures). Currently the government's involvement with development and production of space systems is one of oversight and guidance, marked with very specific requests for capabilities in non-uniform timeframes. In addition, many actors from both the public and private sectors are wary of repeating past mistakes, such as those of the last two decades which have led to huge delays and cost overruns, especially within the defense sector. ${ }^{41}$ This all too often resulted in the government paying upfront for capabilities that contractors then failed to deliver. However, it should be noted that space has now reached a level of maturity where venture capital is willing to take on the risk of developing space systems in exchange for payment-upon-delivery or even the awarding of a long-term services contract. NASA especially has had considerable success contracting out its servicing missions to 
the International Space Station under the Space Act Agreements, and this contract vehicle may serve as a path to contracting other activities as well. ${ }^{5}$

The underlying premise of increased commercialization of space is that risk is shifted from the government to the contractor in exchange for possible return on investment commensurate with other high-risk activities performed in the private sector. Of course, there are still some core activities which would be deemed too expensive, risky, or vital to security to be transferred to the private sector, but several activities, like space weather, terrestrial weather, high-bandwidth communications, expendable launch, and even precision navigation and timing, are now at the point in their technological maturation that private industry would be willing to accept all of the known risks in the pursuit of profits. Furthermore, decreased cost to the government should be attainable under this business model, as it will further enable the government to focus on its core competencies of science, exploration, and national security while offloading responsibility to corporations for tasks that have become well defined over the last decade. For instance, such activities as unmanned launch, low Earth orbit cargo delivery, and fabrication of interchangeable components have all reached a level of understanding such that commercial firms are willing to take on these tasks.

\begin{tabular}{|l|l|}
\hline \multicolumn{2}{|c|}{ Ilities and Attributes Offered Through Commercialization } \\
\hline Robustness & $\begin{array}{l}\text { Commercially hosted payloads allow a system to gain robustness through regular replacement of assets with } \\
\text { more advanced, context-specific payloads, due to the fact that they can be aligned with the typically much } \\
\text { faster replenishment cycle of commercial assets. }\end{array}$ \\
\hline Scalability & $\begin{array}{l}\text { Commercially hosted payloads take advantage of industry standardization practices in order to add } \\
\text { additional components without increasing system complexity. }\end{array}$ \\
\hline Extensibility & $\begin{array}{l}\text { Regular replacement of commercially hosted payloads ensures that capabilities can be upgraded without } \\
\text { major alterations to the overall architecture. }\end{array}$ \\
\hline Testability & $\begin{array}{l}\text { Profit-seeking firms excel at producing standardized, streamlined products, and as such the entire product } \\
\text { line can be more easily tested for quality than a one-time government-funded endeavor. In addition, } \\
\text { commercially-hosted payloads provide a reliable, standardized, and operationally realistic means of testing } \\
\text { new payloads while transferring the risks associated with launch and deployment to commercial firms. }\end{array}$ \\
\hline Quality & $\begin{array}{l}\text { By executing performance and milestone driven contracts, quality can be ensured simply by withholding } \\
\text { payment from firms which do not meet the key performance parameters outlined in the contracts. }\end{array}$ \\
\hline Affordability & $\begin{array}{l}\text { By leveraging competition between firms and allowing industry to provide solutions rather than dictate pre- } \\
\text { determined ones, the commercialization concept should decrease costs throughout system lifecycles and in } \\
\text { the face of shifting economic, political, and technical realities. }\end{array}$ \\
\hline
\end{tabular}

Even with all of the advantages of commercialization, a balance must still be struck between oversight, award of contract, and involvement of proper regulatory agencies. It must be ensured that even as risks are shifted from civil agencies to commercial firms, quality does not suffer due to lack of oversight or unduly cutthroat competition. An additional note is that the barriers to entry in the space industry are high due to the difficulty of launch and the necessary government oversight, but ultimately the lowering of these barriers will be critical to increasing activity and making space a more profitable, quality-driven, standardized marketplace.

With regards to system lifecycle phases, commercialization of space architectures will likely cause the greatest changes in how the space industry behaves in the "Engineering and Manufacturing Development" and "Production and Deployment" phases of the space system's lifecycle. This trend is aimed at decreasing costs and development time by aligning DoD and civil timetables with faster industry timetables, which would help to lessen increasing spacecraft costs and lengthening development cycles. It would also have the added bonus of maturing technology faster as smaller, incremental improvements could be tested in each generation and along much more standardized production methods. CHIRP is the prime example of how an operational launch detection technology could be matured while providing capability in between major upgrades to core DoD infrastructure (which would itself be on a much longer timescale). ${ }^{6}$

\section{B. Reductions in launch costs and the development of hybrid or reusable launch systems}

The major goals of launch cost reductions and the development of reusable launch systems for low Earth orbit delivery are simple: save money and decrease architecture response times to catastrophe or failure. As launch represents a massive portion of every space project's total costs (often with launch costing just as much as the satellite itself), significant reductions in the cost of this one mission component could spark major changes to current 
architectural paradigms, as well as decrease barriers to entry and encourage new, ground-breaking, riskier enterprises in space. Lowered launch costs also promise to make space systems significantly more responsive. For example, the U.S. Army's Soldier-Warfighter Operationally Responsive Deployer for Space (SWORDS) program seeks to field the capability of launching nanosatellites within 24 hours of call-up at a cost of \$1 million per launch vehicle. $^{42}$ In addition, the development of an economically viable and quickly deployable reusable launch system would also radically alter the way space systems engineers think about space vehicle design life and constellation lifecycle design. For example, the goal of DARPA's Airborne Launch Assist Space Access (ALASA) program is to place a $100 \mathrm{lb}$. payload into low Earth orbit at one-third the cost of current expendable launch vehicles. ${ }^{43}$ Such a capability would not only encourage new ventures in space but would also greatly increase the overall responsiveness of space systems.

\begin{tabular}{|l|l|}
\hline \multicolumn{2}{|l|}{ Ilities and Attributes Offered Through Launch Cost Reductions and New Vehicle Development } \\
\hline Robustness & $\begin{array}{l}\text { Lower launch costs and a reusable launch capability would make regular replenishment and upgrades of space } \\
\text { architectures much more economically feasible. The responsiveness of a program like ALASA especially } \\
\text { would allow space architectures to react to context shifts in a matter of days rather than months or years. }\end{array}$ \\
\hline Resiliency & $\begin{array}{l}\text { In the event of catastrophic failure or disruption of space assets, lower launch costs and reusable launch would } \\
\text { enable space architectures to quickly and economically reconstitute constellations. }\end{array}$ \\
\hline Testability & $\begin{array}{l}\text { Less expensive launch and reusable launch would remove one of the primary hurdles to testing new space } \\
\text { systems in an operationally realistic environment, allowing for faster technology maturation through a more } \\
\text { economically efficient means of testing new technologies and concepts. }\end{array}$ \\
\hline Qlexibility & $\begin{array}{l}\text { Easing the currently prohibitive costs of delivering assets to low Earth orbit would add a great deal of } \\
\text { flexibility to space architectures, as they could be designed for replenishment on a much shorter timeframe } \\
\text { with more technologically advanced and context-specific assets. }\end{array}$ \\
\hline Affordability & $\begin{array}{l}\text { While the orbital assets themselves would not necessarily see any gains in performance, the fact that launch is } \\
\text { less expensive should theoretically lead to more launches per year. A second-order consequence of this would } \\
\text { be that, with more launches, the launch vehicles themselves should become more reliable and higher- } \\
\text { performing. }\end{array}$ \\
\hline $\begin{array}{l}\text { Not only does this trend decrease overall system costs, but it allows for the concept of affordable short } \\
\text { timeframe replenishment of assets. Such a concept would be almost unthinkable given the price of current } \\
\text { expendable launch vehicles. }\end{array}$ \\
\hline
\end{tabular}

Decreased launch costs and reusable launch would go far in reducing a primary risk currently facing space architectures: that of the increasingly prohibitive cost of launch adversely effecting architecture development and planning. While large projects launching assets beyond LEO might be able to stomach high launch costs, there are many smaller enterprises which may be turned away from engaging in space activities simply due to the high cost of launch. Creating a more economical means of high-volume small payload delivery to LEO would allow smaller entities the ability to mature technologies and concepts as well as provide steady, reliable business to the space industrial base. In addition, this trend helps to alleviate the risk of being unable to reconstitute an architecture after catastrophe or disruption, simply due to insufficient funding to launch new assets. Reusable launch especially should allow space architecture planners to include a responsive reconstitution capability, allowing for replenishment of assets in a matter of days rather than months or years.

This trend would primarily affect the conduct of the "Production and Deployment" and the "Operations and Support” phases of a space system's lifecycle, as the deployment of assets would become cheaper and more responsive and because systems engineers would have viable contingency options during operations. Rather than design and manufacture space vehicles such that each individual vehicle is survivable, resilient, and flexible for 15+ years, engineers could instead plan for more advanced, more responsive space architectures with assets that are less robust but are also easy to replace with more advanced vehicles every 2-3 years. Large missions would still be able to benefit through the ability to test critical improvements within the current generation, enabling better upgrade to technology without great risk. In addition, this trend is one that could act as a catalyst for the other trends; that is, less expensive launch options would serve to make all other new concepts and technologies easier to test and deploy. 


\section{On-orbit servicing and infrastructure}

The desire for an on-orbit servicing capability is born out of a desire for increased architectural flexibility and resiliency. While manipulating and changing the components of an orbiting asset is likely to always be a difficult endeavor, the ability to do so reliably and within a responsive timeframe is another paradigm-changing capabilityone which could end the accepted doctrine of no further modification of assets once they are in orbit. Furthermore, developing a more robust on-orbit infrastructure would also change the way space architectures are designed and managed, as concepts such as shared power generation and data processing granted by such an infrastructure would open up many new possibilities for architecture design.

\begin{tabular}{|l|l|}
\hline \multicolumn{2}{|c|}{ Ilities and Attributes Offered Through On-Orbit Servicing and Infrastructure } \\
\hline Robustness & $\begin{array}{l}\text { An on-orbit servicing (OOS) capability naturally lends itself to increasing the robustness of space architectures, } \\
\text { as it would allow orbital assets to be upgraded or reconfigured to better perform under context shifts. An } \\
\text { orbital infrastructure would also contribute to robustness, provided that the infrastructure is designed to easily } \\
\text { allow for new components and missions throughout the system's lifecycle. }\end{array}$ \\
\hline Resiliency & $\begin{array}{l}\text { Previously mission-ending catastrophes such as propellant depletion or insertion into an incorrect orbit would } \\
\text { be far easier to correct given a reliable and cost-effective on-orbit servicing capability. Also, a well-designed } \\
\text { infrastructure would prevent a single-point failure in a space system from ending the entire architecture's } \\
\text { mission, and would even allow other assets to replace the capabilities lost due to an individual failure. }\end{array}$ \\
\hline Scalability & $\begin{array}{l}\text { The on-orbit infrastructure concept would grant significant scalability to space architectures, as it would allow } \\
\text { new components and capabilities to be added to an architecture without a corresponding increase in system } \\
\text { complexity, similar to how the Internet scales well with increases in applications and users. }\end{array}$ \\
\hline Extensibility & $\begin{array}{l}\text { The nature of an extensive infrastructure project is that it supports new uses and capabilities without requiring } \\
\text { major changes to system components and the overall architecture. A well-designed on-orbit infrastructure } \\
\text { would include the necessary mechanisms to add capabilities to a space architecture without significant } \\
\text { redesigns of the architecture itself. }\end{array}$ \\
\hline Flexibility & $\begin{array}{l}\text { Rather than launch a space vehicle with no plans for future maintenance, space systems engineers could design } \\
\text { vehicles such that they can be upgraded and maintained periodically, increasing their ability to respond to } \\
\text { context shifts throughout their lifecycle. The best example of this is the Hubble Space Telescope's increases in } \\
\text { performance throughout its lifecycle due to its pre-planned accessibility and upgradeable back-end modules. }\end{array}$ \\
\hline
\end{tabular}

This trend primarily reduces the risk of total mission failure in the event of catastrophe, as assets could be designed such that they are recoverable and even upgradeable with new hardware. Also, the concept of a robust inorbit infrastructure mitigates the risk of a space architecture not having the required power or date processing capability for a mission, as it could "borrow" such commodities from other spacecraft within the infrastructure. A well-designed infrastructure could even enable lower latency for communication and more even power consumption among constellations. However, one risk that this trend might invite would be that a single disruption or bug could work its way through the entire infrastructure, causing many more space vehicles and constellations to be affected than if they were all separate (however, obscurity does not necessarily equal security, and disparate systems today are not safe simply because they are not linked).

The on-orbit servicing and infrastructure trend would most affect the "Material Solution Analysis", "Technology Development," and "Operations and Support" lifecycle phases. As mentioned above, the ability to service or modify space vehicles in orbit would radically alter the current paradigm of near-complete inability to alter a spacecraft from its original mission once it has reached orbit, and not only would this be reflected in operations but it would also change the way space vehicles and architectures are designed (for example, spacecraft could be engineered with the specific ability to be periodically refueled throughout their operational lifecycles). Architectures could also be designed and operated under a paradigm of routine hardware upgrades and the reuse of critical components, such as large apertures. Finally, an on-orbit infrastructure could make large changes in architecture design as well, due to the ability to launch new types of space architectures and constellations which could take full advantage of the infrastructure.

\section{Aggregation and disaggregation of orbital assets}

Aggregation of assets involves the relatively simple act of combining missions on to fewer platforms. While this does act to decrease costs and contribute a degree of affordability to the architecture, aggregation is not viewed as adding any of the other ilities to space systems. In fact, high aggregation of assets may actually decrease the 
resiliency and survivability of the overall architecture, as several missions embarked on a small number of platforms would be more susceptible to catastrophic events or component failures. In addition, a very aggregated architecture would also be lacking in flexibility and robustness, as large spacecraft serving several missions would most likely require longer development and be designed to last much longer in orbit.

On the other end of the spectrum, one of the main goals of a disaggregated space architecture is to reduce the risk of an individual satellite failure substantially degrading an entire mission. Combined with the aggregation of seemingly unrelated missions with similar requirements, the entire architecture can benefit from resource sharing arrangements while simultaneously disaggregating total capability across multiple platforms (e.g. two missions each with two satellites could become one mission with four). Thus, this trend encourages the creation of many smaller space vehicles, which in turn would drive a more robust industrial base while also speeding technological maturation of each small asset. Eventually, this paradigm proposes to cut costs as the assets become more standardized and easier to produce while at the same time lowering barriers to entry to new firms. In addition, aggregated/disaggregated space architectures act in much the same way as an on-orbit infrastructure, in that each small space vehicle is able to contribute to an overall more powerful and capable constellation. Through aggregation or disaggregation of missions across space-based platforms, architectures may shift the approach to mission satisfaction risk posturing as opposed to individual satellite availability.

It is important to note that a majority of the emphasis on smaller disaggregated missions are those that occupy Low Earth Orbit (LEO). However, there are opportunities for this trend to be employed in MEO as well as GEO. CHIRP is one example of the aggregation of a launch detection mission by embarking it aboard a communications mission. ${ }^{6}$ GPS and nuclear detection are another example in MEO where similar mission requirements (specifically, timing) lead to the aggregation of two distinct missions. This is not to say that changes in aggregation are appropriate for all missions, but there do appear to be many missions that possess the right circumstances for this concept to be effective at various stages of their lifecycles, even if it is only in development, maturation, or augmentation. It should also be noted that this activity interplays heavily with commercialization of space and delivery of services as opposed to orbital assets alone. This trend will also likely rely on more automated ground operations and cheaper launch costs as disaggregation implies the creation and launch of more busses and specific payloads. As each satellite is also a communications system, a high number of disaggregated satellites may even start to form an underlying infrastructure for other missions to utilize.

\begin{tabular}{|l|l|}
\hline \multicolumn{2}{|c|}{ Ilities and Attributes Offered Through Disaggregation and Aggregation of Assets } \\
\hline Survivability & $\begin{array}{l}\text { More targets inherently allow a space architecture to degrade gracefully under short duration disturbances. } \\
\text { Furthermore, as the number of assets in a constellation increases, there is less of a probability that a } \\
\text { perturbation will disrupt the entire mission. }\end{array}$ \\
\hline Resiliency & $\begin{array}{l}\text { Permanent loss of one asset will not significantly decrease the overall capability of a disaggregated system. } \\
\text { These systems would also be designed with shorter lifecycles in mind, such that the ability to replenish lost } \\
\text { assets would already be planned within appropriate timeframes (which could also be more easily adjusted). }\end{array}$ \\
\hline Robustness & $\begin{array}{l}\text { Many small, simple, disaggregated payloads can be planned for faster replenishment and upgrade by more } \\
\text { advanced payloads, allowing the entire architecture to adapt to context shifts throughout the system lifecycle. }\end{array}$ \\
\hline Scalability & $\begin{array}{l}\text { An essential aspect to a disaggregated architecture, any system attempting to field a larger number of assets } \\
\text { must be designed to grow and be able to handle the addition of new components. }\end{array}$ \\
\hline Extensibility & $\begin{array}{l}\text { Theoretically, a disaggregated system properly designed to scale up over time would be extensible. New } \\
\text { payloads, capabilities, or whole assets could be added with the interfaces for both payload to bus and bus to } \\
\text { ground abstracted for easy integration. }\end{array}$ \\
\hline Festability & $\begin{array}{l}\text { Disaggregated architectures enable lower-risk testing as well as much simpler space vehicles with fewer } \\
\text { failure points. More launches and space activities overall across the disaggregated architecture would also } \\
\text { enable incremental improvement as well as incremental testing as new components are added. }\end{array}$ \\
\hline
\end{tabular}

Because the disaggregated space architecture trend is aimed at reducing overall risks to the system, there are few inherent risks that this trend invites beyond the chance that a common failure point is found to disrupt all vehicles in

14

American Institute of Aeronautics and Astronautics 
the constellation. The fact that the architecture is composed of many smaller, simpler space vehicles would theoretically allow for a responsive replenishment of assets in the face of catastrophe or disruption. The disaggregated concept would also mitigate the risk of orbital assets becoming technologically obsolete or not being appropriate to a mission after a context shift. Proponents of largely disaggregated architectures theorize that with common requirements and interfaces, entire constellations would share enough similarities such that each generation would improve on the last. A good example of such a case would be cellular phones, which in recent years have built upon the operating system and hardware of the last generation while still working within the same infrastructure and deflecting different threats to the system.

This trend's paradigm changes would most likely be felt in the "Technology Development" and "Engineering and Manufacturing Development” phases of the system lifecycle, as systems engineers would have to dramatically alter the architectural design from one of a few monolithic space vehicles to one of large constellations of many small satellites. Also, the space industrial base would stand to greatly benefit from this paradigm shift, as technologies could be matured faster and standardized much more easily across many small space vehicles. It should be noted that to date no material solution analysis has identified a disaggregated constellation as an appropriate way of executing a mission. However, it has only been in the last five years that technology has even enabled this possibility at a reasonable price point.

\section{E. Automation and standardization of ground architectures}

The benefits of automated ground systems are currently two-fold. First, a more standardized and automated ground architecture would add extensibility and scalability to space architectures. Second, automated operations should allow for more robust, affordable, and flexible systems within day-to-day operations. This is because in general, abstraction of interface leads to scalability, with each new satellite added to an automated common system not altering the underlying software or human interface; instead, all commands are abstracted as database inputs. This scalability component also allows the system to remain affordable as more assets are added and the operating locations of the ground stations change. Properly designed, this allows the system to be extensible as well, in that the underlying logic engine responsible for allocating resources to satellites does not change. Indeed, it does not even need to be thought of as a space system but rather as a simple logic solver.

Flexibility in overcoming short duration problems is also granted by the common automated ground architecture concept. As noted by Cutler, much of the scheduling at AFSCN is performed manually, requiring substantial and unnecessary manpower. ${ }^{44}$ Under an automated ground architecture, the loss of a pass or even a component (e.g. multiplexer, dish, encoder, etc.) can be recomputed for optimal realignment (or degradation if needed) within a matter of milliseconds. Existing systems currently cannot change a 24-hour schedule once it is in place, but an automated logic engine copes with any change within milliseconds. Indeed, it is now well known that computers are typically a good replacement for humans when a problem can be abstracted to rules and values for satisfaction of these rules. As such, automated ground systems are better suited to satisfying the needs of satellite contacts. After the computer on the ground has made its calculations, a human could then approve the rule set or tell the computer to re-compute, with different parameters, if the answer is unsatisfactory. This can also negate the need for full around-the-clock staffing of ground stations, making constellations more affordable to keep on orbit. Furthermore, in the event of an on-orbit anomaly, CGA can automatically safe a vehicle or follow any checklist that would be employed by a human operator, much more quickly and without mistake. ${ }^{45}$ For unique anomalies, senior technicians will still be required, but in the current paradigm they are already on call and not on regular rotation anyway. Thus, this trend substantially changes the way space operations would be conducted. 


\begin{tabular}{|c|c|}
\hline \multicolumn{2}{|r|}{ Ilities and Attributes Offered Through Automated Ground Architecture } \\
\hline Survivability & $\begin{array}{l}\text { The system would compute all passes for each 24-hour period and reassign all assets to achieve maximum } \\
\text { utility in the event of disruptions/perturbations. The system could also automatically safe satellites in the event } \\
\text { of disruption, allowing for faster recovery from known failure modes. }\end{array}$ \\
\hline Scalability & $\begin{array}{l}\text { Each node is designed to handle a set number of space vehicles, with capacity increased by simply adding } \\
\text { nodes and scaling linearly. Network capacity could also grow by adding new sites or by adding more } \\
\text { hardware at existing sites. }\end{array}$ \\
\hline Extensibility & $\begin{array}{l}\text { Each node would operate independently but use peer-to-peer contacts to share available resources on a non- } \\
\text { conflicting basis. The system can also add more sites as well as more satellites by increasing hardware or by } \\
\text { adding new command and telemetry databases. }\end{array}$ \\
\hline Testability & $\begin{array}{l}\text { On site/at factory testing would be performed using an actual operational node ensuring compatibility between } \\
\text { the space vehicle and the ground logic. The size of test assets could also be reduced even as availability of } \\
\text { testing is increased. }\end{array}$ \\
\hline Flexibility & $\begin{array}{l}\text { Addition of new assets to operations would be easier; for instance, changing the priorities of contacts would } \\
\text { not depend on past work. Space vehicle designers could also create their own database for command and } \\
\text { telemetry, with hardcoding and compiling not required as such integration would be instant. }\end{array}$ \\
\hline Affordability & $\begin{array}{l}\text { Automation reduces the number of human satellite operators and planners required. Testing would also only } \\
\text { require one rack as opposed to an entire tractor-trailer (of which typically only one exists). }\end{array}$ \\
\hline
\end{tabular}

One example illustrating the flexibility granted by automating ground architectures is Tiger Innovations' work on STPSat-1 at NRL's Blossom Point satellite tracking facility. Virtually all of STPSat-1's operational activities after launch and early orbit checkout were executed automatically by the CGA. ${ }^{46}$ STPSat- 1 was then able to quickly integrate into the existing manifest of daily contacts, and all command and control as well as mission data was processed through the CGA and sent appropriately for further processing. The Air Force's SENSE mission also made use of CGA as a rapid prototyping tool to both test the system's end-to-end functionality before launch as well as virtually "fly" the engineering models on the ground before launch. The system was able to seamlessly integrate all commands required by the SENSE mission in its command and telemetry database. ${ }^{23}$ SENSE was also granted access to AFSCN; while ultimately successful due to high quality personnel and efficient operational practices, it was found that it was much more difficult to integrate with AFSCN than with CGA. These two examples both illustrate how automated ground operations play a role in reducing both costs and risks by adding ease of testability for new systems.

The changes to architectures brought about by more standardized, automated ground systems would primarily be felt in the "Engineering and Manufacturing Development" and "Operations and Support" phases of architecture development. The former phase would be affected in that new spacecraft would have to be designed such that they can interact seamlessly with new ground architectures; while this is not viewed as technically or economically prohibitive, it would likely require a high degree of coordination amongst various manufacturers and spacecraft operators. The "Operations and Support" phase, though, would experience several positive changes, including a decrease in manpower required for daily operation of constellations and easier integration of new space vehicles into the overall architecture.

\section{Conclusions and Future Work}

While these five trends have now been justified as potentially paradigm-changing concepts in space architectures, there is still much research and testing to be done in order to determine the full value that each may unlock in future architectures. The two basic scenarios under which the ilities offered by each trend must still be tested are normal and abnormal operations. For commercial and civil stakeholders, the most important case will be improvements under normal operations; in other words, under such conditions as can be reasonably extrapolated from the current operational environment. While testing under normal conditions is also important to DoD architectures, these systems also must possess the needed ilities to operate under the potentially radical context shifts brought about by military operations. The careful balance of building affordable systems that deliver the desired performance and ilities must be examined under both these contexts, and the structure outlined in this paper will serve as foundation for this examination. 
Another key area of future research will be the effect that these five change trends would have on the economic feedback loops which characterize the aerospace industry. One dominating trend of recent decades has been the development of systems which are more reliable and longer-lasting, which in turn means that fewer systems are produced and launched each year. A second order consequence of this is a loop of increasing costs and lengthening development times (as no one wants expensive, reliable, long-lasting systems to fail during delivery), which in turn weakens the industrial base, as space vehicle manufacturers struggle to innovate or turn a profit when the technologies being developed follow a decade-old plan. Many of these trends provide a means of reversing this trend and spurring greater entrepreneurial activity. Disaggregation and on-orbit servicing especially suggest that making more individual spacecraft with shorter life spans, less individual reliability, and planned maintenance and upgrades will actually decrease life-cycle cost over time. This way of thinking also shifts the risk posture from one of individual monolithic satellites to that of overall life-cycle cost and maintaining of a service, rather than a single piece of hardware.

Several tools are available to explore these possible trade-spaces and evaluate the potential value of each ility proposed by these five trends. Ultimately, a business case must be made before implementing any policy or program which seeks to exploit these ideas, driven by the testing of these ilities and trends using trade-space exploration and other means of demonstrating the value of these designs under context shift. To perform this work the authors propose the use of system dynamics models in conjunction with epoch-era analysis to determine overall utility of potential architectural decisions-first under the conditions that can be reasonably expected in the current space environment, and then under context shifts. Rule-based approaches should be a main focus of future work, and the use of satellite orbit modeling for proposed architectures will also be essential in validating key performance parameters such as revisit times and ground coverage offered by many different systems. Of course, to believe a model of this caliber the results must align with physical reality, and what makes this type of evolutionary modeling especially difficult is that these systems cannot be designed from scratch. They must start with existing capabilities and then plot how to get to a new system without degrading current capabilities or breaking cost limits.

It is our belief that further analysis and operational testing of these five paradigm-changing trendscommercialization, launch cost reductions and reusable launch, on-orbit servicing and infrastructure, disaggregation and aggregation of assets, and automated ground architectures-will ultimately lead to not only new technologies and architectural paradigms but also new policies aimed at unlocking an entrepreneurial loop in space and the lowering of barriers to entry in the space industry. With increasing costs and lengthening development cycles threatening the very core of the space industrial base, it is increasingly vital that one or more of these trends, or another yet unidentified, must be implemented to restructure the technological, political, and acquisitions paradigms which drive future space architectures. 


\section{References}

${ }^{1}$ Anderson, Paul R., and Lucy Bartamian. "Growth Trends in Communication Satellites and the Impact on Satellite System Architecture." 26th International Communications Satellite Systems Conference (ICSSC), 2008.

${ }^{2}$ Office of the Under Secretary of Defense for Acquisition, Technology, and Logistics. "Report of the Defense Science Board/Air Force Scientific Advisory Board Joint Task Force on Acquisition of National Security Space Programs." May 2003.

${ }^{3}$ Figliola, Patricia, Carl Behrens, and Daniel Morgan. U.S. Space Programs: Civilian, Military, and Commercial. Congressional Research Service, 2006.

${ }^{4}$ Air Force Space Command. "Resiliency and Disaggregated Space Architectures." White Paper, 2013.

${ }^{5}$ Anderson, Chad. "Rethinking public-private space travel." Space Policy, 2013: 266-271.

${ }^{6}$ Gong, R., M. Broder, L. Jocic, and J. Gee. "Space Architecture Transition with Hosted Payloads." AIAA Space $2012,2012$.

${ }^{7}$ Meyer, Robinson. "Silicon Valley's New Spy Satellites." The Atlantic. Jan 14, 2014. http://www.theatlantic.com/technology/archive/2014/01/silicon-valleys-new-spy-satellites/282580/.

${ }^{8}$ Pomerantz, William J., and Steven J. Isakowitz. "Virgin Galactic." New Space, 2013: 167-170.

${ }^{9}$ Rasky, D., R. Pittman, and M. Newfield. "The Reusable Launch Vehicle Challenge." AIAA Space 2006, 2006.

${ }^{10}$ Bartolotta, P., A. Wilhite, M. Schaffer, L. Huebner, R. Voland, and D. Voracek. "Near-term Horizontal Launch for Flexible Operations: Results of the DARPA/NASA Horizontal Launch Study." AIAA Space 2012, 2012.

${ }^{11}$ Space Exploration Technologies. Capabilities and Services. 2013. http://www.spacex.com/about/capabilities.

${ }^{12}$ Richards, M. "On-Orbit Serviceability of Space System Architectures." SM Thesis, 2004.

${ }^{13}$ Long, A., M. Richards, and D. Hastings. "On-Orbit Servicing: A New Value Proposition for Satellite Design and Operation." Journal of Spacecraft and Rockets, 2007.

${ }^{14}$ Benedict, B. "Rationale for Need of In-Orbit Servicing Capabilities for GEO Spacecraft." AIAA Space 2013, 2013.

${ }^{15}$ Hubble Site. Team Hubble: Servicing Missions. 2013. http://hubblesite.org/the_telescope/team_hubble/servicing_missions.php.

${ }^{16}$ Golkar, Alessandro. "Federated Satellite Systems (FSS): A Vision Towards an Innovation in Space Systems Design." IAA Symposium on Small Satellites for Earth Observation, 2013.

${ }^{17}$ Golkar, Alessandro. "Federated Satellite Systems: A Case Study on Sustainability Enhancement of Space Exploration Systems Architectures." 64th International Astronautical Congress, Beijing, China, 2013.

${ }^{18}$ Golkar, Alessandro, and Ignasi Lluch. "Satellite-to-Satellite Coverage Optimization Approach for Opportunistic Inter-Satellite Links." IEEE Aerospace Conference, 2013.

${ }^{19}$ Mathieu, C. "Assessing the Fractionated Spacecraft Concept." SM Thesis, 2006.

${ }^{20}$ Mathieu, C., and A. Weigel. "Fractionated Spacecraft Architecture Seeding Study." AFRL Report, 2006.

${ }^{21}$ Brown, O., and P. Eremenko. "Application of Value-Centric Design to Space Architectures: The Case of Fractionated Spacecraft." DARPA Report, 2008.

${ }^{22}$ Sondecker, George, Paul La Tour, and Lyle Abramowitz. "SENSE: The USAF SMC/XR NanoSatellite Program for Space Environmental Monitoring." 27th Annual AIAA/USU Conference on Small Satellites, 2013.

${ }^{23}$ La Tour, Paul, George Sondecker, Jonwa Kim, and Lyle Abramowitz. "U.S. Air Force's SMC/XR Space Environmental NanoSat Experiment." AIAA Space 2012 Conference \& Exposition, 2012.

${ }^{24}$ Jun Tominaga, Jose Demisio Simoes da Silva, Mauricio Goncalves Vieira Ferreira. "A Proposal for Implementing Automation in Satellite Control Planning." Space Ops 2008 Conference. AIAA, 2008.

${ }^{25}$ Laura Barbulescu, Adelle Howe, Darrell Witley. "AFSCN scheduling: How the problem and solution have evolved." Mathematical and computer Modeling, 2006: 1023-1037.

${ }^{26}$ Griffith, Robert. Mobile Cubesat Command and Control (MC3). Thesis, Montery, CA: Naval Postgraduate School, 2011.

${ }^{27}$ NASA Office of Safety and Mission Assurance. “Agency Risk Management Procedural Requirements.” NASA Procedural Requirements, 2008.

${ }^{28}$ Szajnfarber, Z., Richards, M., and Weigel, A. "Implications of DoD Acquisition Policy for Innovation: The Case of Operationally Responsive Space.” AIAA Space 2008, 2008.

${ }^{29}$ Cross, Robert. "Estimating the Risk of a New Launch Vehicle Using Historical Design Element Data.” International Journal of Performability Engineering, Vol. 9, No. 6, pp. 599-608, November 2013.

${ }^{30}$ Murman, E., Walton, M., and Rebentisch, E. “Challenges in the Better, Faster, Cheaper Era of Aeronautical Design, Engineering, and Manufacturing.” Lean Aerospace Initiative Report Series, September 2000.

${ }^{31}$ de Weck, Olivier, Daniel Roos, and Christopher Magee. Engineering Systems: Meeting Human Needs in a Complex Technological World. Cambridge: MIT Press, 2011. 
${ }^{32}$ de Weck, Olivier, Adam Ross, and Donna Rhodes. "Investigating Relationships and Semantic Sets amongst System Lifecycle Properties (Ilities)." Third International Engineering Systems Symposium, CESUN 2012, Delft University of Technology, 2012.

${ }^{33}$ Ross, Adam, and Donna Rhodes. "Using Natural Value-Centric Time Scales for Conceptualizing System Timelines through Epoch-Era Analysis." INCOSE International Symposium 2008, June 2008.

${ }^{34}$ Schaffner, Michael, Marcus Wu, Adam Ross, and Donna Rhodes. "Enabling Design for Affordability: An Epoch-Era Analysis Approach." NPS Tenth Annual Acquisition Research Symposium Acquisition Management. 2013.

${ }^{35}$ Richards, Matthew, Adam Ross, Daniel Hastings, and Donna Rhodes. "Two Empirical Tests of Design Principles for Survivable System Architecture." INCOSE International Symposium, 2008.

${ }^{36}$ Beesemyer, Jay. "Empirically Characterizing Evolvability and Changeability in Engineering Systems." SM Thesis, 2012.

${ }^{37}$ Binder, Robert. "Design for testability in object-oriented systems." Communications of the ACM, 1994.

${ }^{38}$ Viscito, L. "Quantifying Flexibility in the Operationally Responsive Space Paradigm." SM Thesis, 2009.

${ }^{39}$ U.S. Air Force Operationally Responsive Space Office. Mission. 2013. http://ors.csd.disa.mil/mission/.

${ }^{40}$ Department of Defense Directive (DoDD) 5000.01. “The Defense Acquisition System.” Defense Acquisition Guidebook. Accessed 2014. https://acc.dau.mil/communitybrowser.aspx?id=527180.

${ }^{41}$ Chaplain, Christina T. Space Acquisitions: Acquisition Management Continues to Improve but Challenges Persist for Current and Future Programs. Testimony, Washington D.C.: United States Government Accountability Office, 2014.

${ }^{42}$ U.S. Army Space \& Missile Defense Command/Army Forces Strategic Command. SWORDS: Soldier-Warfighter Operationally Responsive Deployer for Space. 2013.

${ }^{43}$ DARPA Tactical Technology Office. Airborne Launch Assist Space Access (ALASA). 2011.

${ }^{44}$ James Cutler, Armando Fox. "A Framework for Robust and Flexible Ground Station Networks." Journal of Aerospace Computing, Information, and Communications, 2006: Vol. 3.

${ }^{45}$ Klein, Patricia. Naval Research Laboratory Automated Ground Operations. May 2010.

${ }^{46}$ Doug Firestone, Robert Atkin, Carl Hooks, Christoph Englert, David Sisking, Paul Bernhardt, Carl Siefring, Patricia Klein. "STP-Sat1 Automated Ground Operations; Low-Cost, Automated Ground Station for LEO Mission Support." IEEE A\&E Systems Magazine (Tiger Innovations), 2011. 